Supplementary material for

\title{
Structure, Binding Energies, and IR-spectral Fingerprinting of Formic acid Dimers
}

\author{
Ilhan Yavuz
}

Physics Department, Marmara University, Istanbul Turkey

Carl Trindle

The University of Virginia, Charlottesville VA 22904 USA 
Table S1: Monomeric Formic acid in MP2/6-311+G(d,p)

\begin{tabular}{|l|c|c|c|c|}
\hline Species I & Monomer cis & $\begin{array}{c}\text { Monomer } \\
\text { trans }\end{array}$ & Trans. State & $\begin{array}{c}\text { Energy difference } \\
\text { (activation energy) }\end{array}$ \\
\hline Energy & $\begin{array}{c}-189.3547388 \\
\text { Hartrees }\end{array}$ & $\begin{array}{c}-189.3621444 \\
\text { Hartrees }\end{array}$ & $\begin{array}{c}-189.3411260 \\
\text { Hartrees }\end{array}$ & $\begin{array}{c}4.65 \mathrm{kcal} / \mathrm{mol} \\
(13.18 \mathrm{kcal} / \mathrm{mol})\end{array}$ \\
\hline $\mathrm{ZPE}$ & 21.1770 & 21.42957 & 20.10134 & $4.40 \mathrm{kcal} / \mathrm{mol}$ \\
$\mathrm{kcal} / \mathrm{mol}$ & $\mathrm{kcal} / \mathrm{mol}$ & $\mathrm{kcal} / \mathrm{mol}$ & $\mathrm{kcal} / \mathrm{mol}$ & $(11.85 \mathrm{kcal} / \mathrm{mol})$ \\
\hline
\end{tabular}

Table S2: Binding energies and relative energies of dimers of formic acid. Notation of Turi is employed. $\mathrm{D}_{\mathrm{e}}$ in black, $\mathrm{D}_{0}$ (ZPE-corrected values) in bold, Energies in parentheses are in kcal relative to II.

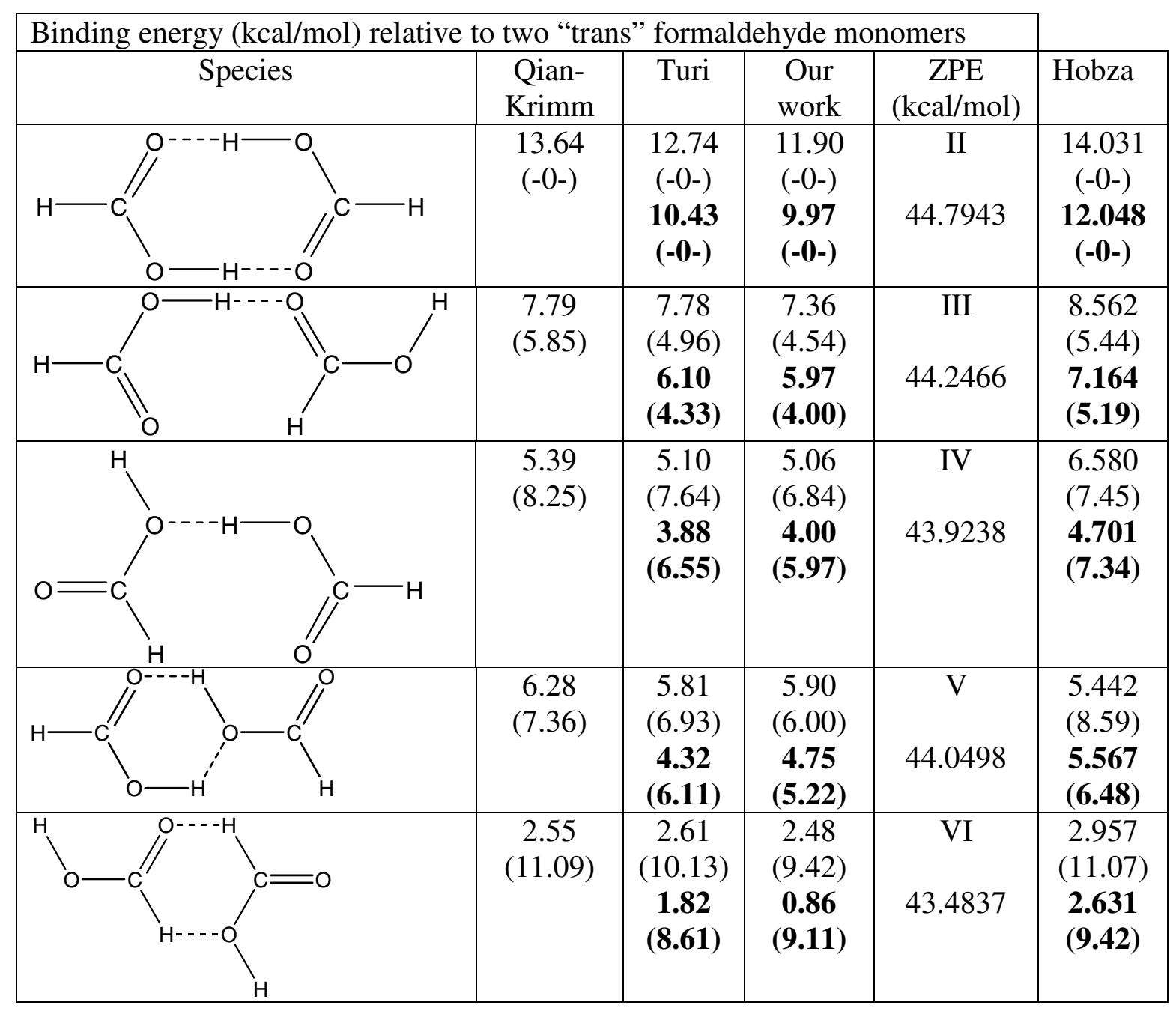




\begin{tabular}{|c|c|c|c|c|c|}
\hline & 1.93 & 1.91 & 1.87 & $\mathrm{VII}$ & \\
\hline $\mathrm{H}$ & $(11.71)$ & $(10.83)$ & $(10.03)$ & & \\
& & $\mathbf{1 . 3 2}$ & $\mathbf{1 . 3 9}$ & 43.340 & \\
& & $\mathbf{( 9 . 1 1 )}$ & $\mathbf{( 8 . 5 8 )}$ & & \\
& & & & & \\
\hline
\end{tabular}

Table S2B: New equilibrium structures and energies relative to (I-trans) $)_{2}=2 \mathrm{~T}$ and Itrans: I-cis $=\mathrm{C}+\mathrm{T}(\mathrm{kcal} / \mathrm{mol})$

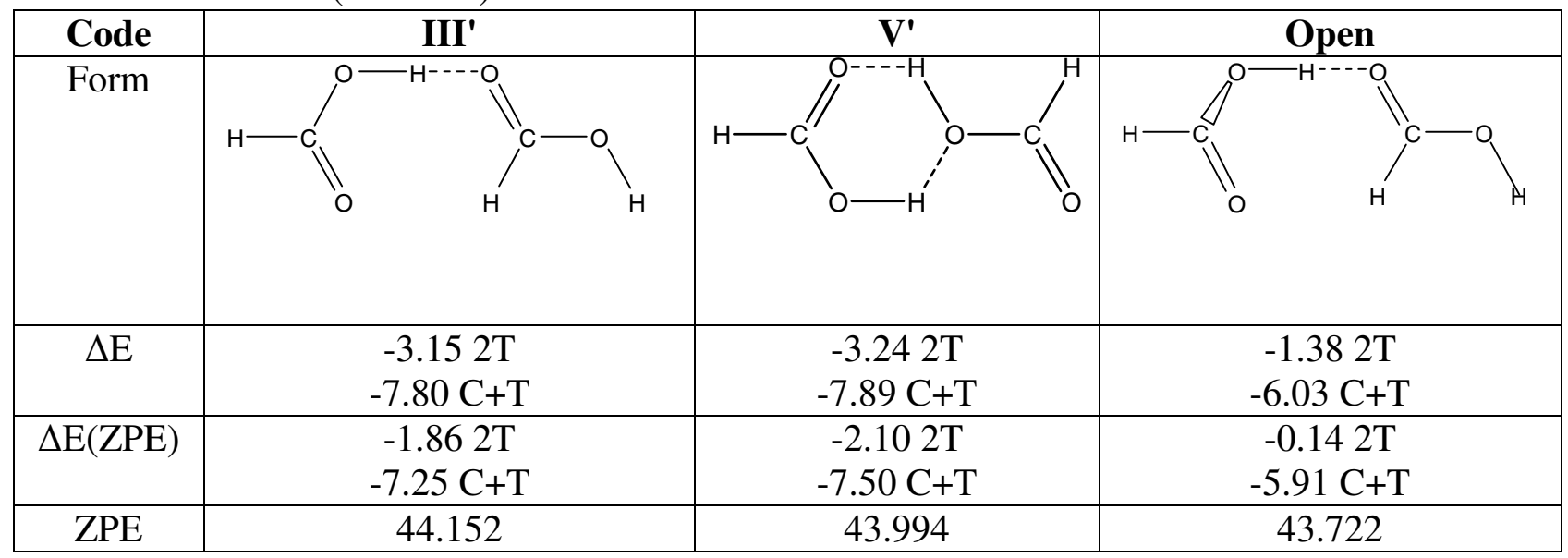

Table S2C: Geometric parameters for Species II - VIII

\begin{tabular}{|c|c|c|c|c|}
\hline Species & I & II & III & IV \\
\hline $\mathrm{C}=\mathrm{O} \quad$ (Turi) & 1.216 & 1.233 & $1.226,1.224$ & $1.217,1.208$ \\
\hline $\mathrm{C}=\mathrm{O} \quad(\mathrm{Y}-\mathrm{T})$ & 1.205 & 1.218 & $1.214,1.212$ & $1.210,1.202$ \\
\hline $\mathrm{C}-\mathrm{O} \quad$ (Turi) & 1.353 & 1.323 & $1.340,1.337$ & $1.338,1.368$ \\
\hline $\mathrm{C}-\mathrm{O} \quad(\mathrm{Y}-\mathrm{T})$ & 1.348 & 1.323 & $1.337,1.334$ & $1.339,1.365$ \\
\hline $\mathrm{OH} \quad$ (Turi) & 0.974 & 0.997 & $0.974,0.980$ & $0.974,0.980$ \\
\hline $\mathrm{OH} \quad(\mathrm{Y}-\mathrm{T})$ & 0.969 & 0.984 & $0.970,0.980$ & $0.969,0.975$ \\
\hline (Turi) & 1.094 & 1.093 & $1.091,1.095$ & $1.091,1.095$ \\
\hline$(\mathrm{Y}-\mathrm{T})$ & 1.096 & 1.095 & $1.093,1.097$ & $1.094,1.097$ \\
\hline$-\mathrm{OH} \ldots \mathrm{O}=$ (Turi) & - & 1.702 & 1.788 & 1.899 \\
\hline$-\mathrm{OH} \ldots \mathrm{O}=(\mathrm{Y}-\mathrm{T})$ & - & 1.816 & 1.895 & 2.000 \\
\hline$-\mathrm{CH} \ldots \mathrm{O}=$ (Turi) & - & - & 2.387 & 2.374 \\
\hline$-\mathrm{CH} \ldots \mathrm{O}=(\mathrm{Y}-\mathrm{T})$ & - & - & 2.493 & 2.538 \\
\hline
\end{tabular}




\begin{tabular}{|c|c|c|c|c|}
\hline Species & $\mathbf{V}$ & VI & VII & VIII \\
\hline $\mathrm{C}=\mathrm{O} \quad$ (Turi) & $1.217,1.208$ & $1.219,1.216$ & 1.216 & 1.220 \\
\hline $\mathrm{C}=\mathrm{O} \quad(\mathrm{Y}-\mathrm{T})$ & $1.212,1.203$ & $1.208,1.205$ & 1.205 & 1.208 \\
\hline $\mathrm{C}-\mathrm{O}$ (Turi) & $1.341,1.358$ & $1.350,1.362$ & 1.360 & 1.351 \\
\hline $\mathrm{C}-\mathrm{O} \quad(\mathrm{Y}-\mathrm{T})$ & $1.338,1.352$ & $1.346,1.356$ & 1.354 & 1.347 \\
\hline $\mathrm{OH}$ (Turi) & $0.978,0.970$ & $0.974,0.974$ & 0.974 & 0.974 \\
\hline $\mathrm{OH} \quad(\mathrm{Y}-\mathrm{T})$ & $0.976,0.973$ & $0.969,0.969$ & 0.969 & 0.969 \\
\hline (Turi) & $1.092,1.088$ & $1.093,1.092$ & 1.093 & 1.092 \\
\hline$(\mathrm{Y}-\mathrm{T})$ & $1.097,1.095$ & $1.095,1.094$ & 1.095 & 1.095 \\
\hline $\begin{array}{l}-\mathrm{OH} \ldots \mathrm{O}< \\
-\mathrm{OH} . . \mathrm{O}=\text { (Turi) }\end{array}$ & $\begin{array}{l}1.963 \\
1.975\end{array}$ & $\begin{array}{l}2.535 \\
2.422\end{array}$ & 2.527 & 2.447 \\
\hline $\begin{array}{l}-\mathrm{CH} \ldots \mathrm{O}< \\
-\mathrm{CH} . . \mathrm{O}=(\mathrm{Y}-\mathrm{T})\end{array}$ & $\begin{array}{l}2.078 \\
2.125\end{array}$ & $\begin{array}{l}2.641 \\
2.535\end{array}$ & 2.619 & 2.569 \\
\hline
\end{tabular}

Table S2D: Selected geometric parameters for cis-trans formic acid dimers (Ang) Geometric parameters are reported for donor (D) and acceptor (A) except for $\mathbf{V}^{\prime}$ ' where this is not easy to define.

\begin{tabular}{|l|c|c|c|}
\hline Species & $\begin{array}{c}\text { III } \\
\text { D, A }\end{array}$ & V' & $\begin{array}{c}\text { OPEN } \\
\text { Trans-donor }\end{array}$ \\
\hline $\mathrm{C}=\mathrm{O} \quad(\mathrm{Y}-\mathrm{T})$ & $1.212,1.203$ & $1.208,1.205$ & $1.210,1.205$ \\
\hline $\mathrm{C}-\mathrm{O} \quad(\mathrm{Y}-\mathrm{T})$ & $1.338,1.352$ & $1.346,1.356$ & $1.338,1.344$ \\
\hline $\mathrm{OH} \quad(\mathrm{Y}-\mathrm{T})$ & $0.976,0.973$ & $0.969,0.969$ & $0.976,0.964$ \\
\hline $\mathrm{CH} \quad(\mathrm{Y}-\mathrm{T})$ & $1.097,1.095$ & $1.095,1.094$ & $1.097,1.100$ \\
\hline$-\mathrm{OH} \ldots \mathrm{O}=$ & 1.864 & 2.015 & 1.983 \\
$-\mathrm{OH} \ldots \mathrm{O}<$ & & 2.055 & \\
$-\mathrm{CH} \ldots \mathrm{O}=(\mathrm{Y}-\mathrm{T})$ & 2.384 & & \\
\hline
\end{tabular}


Table S3: Frequencies for species III

\begin{tabular}{|c|c|c|c|c|}
\hline \multicolumn{2}{|c|}{$\begin{array}{c}\text { Species III MP2/6-311+G(d,p) } \\
\text { Full CP correction }\end{array}$} & Mode & \multicolumn{2}{c|}{$\begin{array}{c}\text { Species III MP2/6-311+G(d,p) } \\
\text { No CP correction }\end{array}$} \\
\hline harmonic & Anharmonic & & harmonic & Anharmonic \\
\hline 3791.2 & 3602.2 & & 3788.9 & 3599.9 \\
\hline 3579.8 & 3396.2 & OH* $^{*}$ str & 3515.0 & 3320.1 \\
\hline 3176.7 & 3028.6 & & 3180.9 & 3031.8 \\
\hline 3121.6 & 2964.3 & & 3119.2 & 2963.0 \\
\hline $\mathbf{1 7 9 8 . 4}$ & $\mathbf{1 7 6 5 . 1}$ & $\mathbf{C = O}$ anti & $\mathbf{1 7 9 5 . 2}$ & $\mathbf{1 7 6 3 . 2}$ \\
\hline 1770.6 & 1739.6 & $\mathrm{C}=$ O sym & 1763.8 & 1735.5 \\
\hline 1448.9 & 1405.9 & & 1456.4 & 1406.4 \\
\hline 1427.0 & 1409.6 & & 1425.0 & 1405.5 \\
\hline 1375.9 & 1330.6 & & 1392.4 & 1348.7 \\
\hline 1332.1 & 1295.3 & & 1335.9 & 1292.9 \\
\hline $\mathbf{1 2 0 5 . 1}$ & $\mathbf{1 1 6 8 . 0}$ & CO donor & $\mathbf{1 2 1 6 . 5}$ & $\mathbf{1 1 7 8 . 7}$ \\
\hline $\mathbf{1 1 6 9 . 4}$ & $\mathbf{1 1 3 5 . 0}$ & CO acceptor & $\mathbf{1 1 7 3 . 5}$ & $\mathbf{1 1 3 9 . 1}$ \\
\hline 670.4 & 660.1 & & 679.9 & 667.2 \\
\hline 648.1 & 640.2 & & 652.5 & 644.5 \\
\hline 153.7 & 140.3 & & 174.7 & 159.0 \\
\hline 135.8 & 126.7 & & 144.3 & 137.4 \\
\hline 95.6 & 82.5 & & 103.7 & 94.0 \\
\hline & & & & \\
\hline 1096.7 & 1062.9 & & 1097.8 & 1070.4 \\
\hline 1078.5 & 1051.0 & & 1080.8 & 1057.6 \\
\hline $\mathbf{8 5 5 . 2}$ & $\mathbf{8 0 6 . 5}$ & OH* oop & $\mathbf{8 7 7 . 0}$ & $\mathbf{8 5 8 . 0}$ \\
\hline $\mathbf{6 9 1 . 1}$ & $\mathbf{6 3 9 . 4}$ & OH oop & $\mathbf{6 8 8 . 5}$ & $\mathbf{6 3 7 . 6}$ \\
\hline 179.3 & 168.6 & & 181.9 & 178.9 \\
\hline 94.6 & 70.2 & & 88.0 & 83.9 \\
\hline 57.4 & 59.5 & & 54.8 & 56.9 \\
\hline
\end{tabular}

Italic $=$ intense absorbers 
Table S4 Frequencies for species IV

\begin{tabular}{|c|c|c|c|c|}
\hline \multicolumn{2}{|c|}{$\begin{array}{c}\text { Species IV MP2/6-311+G(d,p) } \\
\text { Full CP correction/inc. }\end{array}$} & & \multicolumn{2}{c|}{$\begin{array}{c}\text { Species IV MP2/6-311+G(d,p) } \\
\text { No CP correction }\end{array}$} \\
\hline Harmonic & Anharmonic & & Harmonic & Anharmonic \\
\hline 3735.3 & 3545.3 & & 3792.1 & 3605.7 \\
\hline 3631.3 & 3447.2 & & 3658.9 & 3472.7 \\
\hline 3167.4 & 3018.4 & & 3174.7 & 3029.3 \\
\hline 3131.5 & 2956.3 & & 3126.0 & 2968.6 \\
\hline 1813.7 & 1780.2 & $\mathrm{C}=$ O acceptor & 1813.9 & 1780.0 \\
\hline 1798.4 & 1765.8 & $\mathrm{C}=$ O donor & 1793.2 & 1760.4 \\
\hline 1446.8 & 1405.5 & & 1450.1 & 1402.2 \\
\hline 1431.1 & 1405.4 & & 1426.1 & 1395.9 \\
\hline 1365.7 & 1317.8 & & 1378.6 & 1328.3 \\
\hline 1293.4 & 1240.0 & & 1281.8 & 1239.6 \\
\hline $\mathbf{1 1 9 4 . 2}$ & $\mathbf{1 1 5 6 . 2}$ & C-O(-H) donor & $\mathbf{1 1 9 7 . 6}$ & $\mathbf{1 1 6 0 . 3}$ \\
\hline $\mathbf{1 1 1 2 . 5}$ & $\mathbf{1 0 8 0 . 0}$ & C-O(-H) acceptor & $\mathbf{1 0 9 7 . 6}$ & $\mathbf{1 0 6 5 . 7}$ \\
\hline 667.1 & 656.6 & & 676.7 & 642.6 \\
\hline 635.8 & & & 629.7 & 621.3 \\
\hline 132.5 & 120.5 & & 150.6 & 121.4 \\
\hline 125.7 & 117.2 & & 138.1 & 127.5 \\
\hline 85.5 & 74.8 & & 97.1 & 85.1 \\
\hline & & & & \\
\hline 1075.7 & 1048.2 & $19(1)$ & 1075.4 & 1048.5 \\
\hline 1073.2 & 1048.4 & $20(1)$ & 1073.9 & 1043.0 \\
\hline $\mathbf{8 1 9 . 7}$ & $\mathbf{7 6 3 . 1}$ & H out of plane & $\mathbf{8 1 8 . 2}$ & $\mathbf{6 9 2 . 8}$ \\
\hline $\mathbf{6 9 0 . 9}$ & $\mathbf{6 5 6 . 5}$ & H out of plane & $\mathbf{6 5 5 . 5}$ & $\mathbf{5 7 9 . 3}$ \\
\hline 162.6 & 142.2 & $23(1)$ & 155.8 & 111.5 \\
\hline 126.7 & 115.2 & $24(1)$ & 71.1 & 57.2 \\
\hline 45.4 & 35.6 & $18(1)$ & 44.2 & 26.7 \\
\hline & & & & \\
\hline & & & & \\
\hline & & & & \\
\hline
\end{tabular}

Italic $=$ intense absorber 
Table S5

\begin{tabular}{|c|c|c|c|c|}
\hline \multicolumn{2}{|c|}{$\begin{array}{c}\text { Species V MP2/6-311+G(d,p) } \\
\text { Full CP correction }\end{array}$} & & \multicolumn{2}{c|}{$\begin{array}{c}\text { Species V MP2/6-311+G(d,p) } \\
\text { No CP correction }\end{array}$} \\
\hline Harmonic & Anharmonic & & Harmonic & Anharmonic \\
\hline 3723.5 & 3537.3 & & 3693.5 & 3513.1 \\
\hline 3688.0 & 3494.7 & & 3637.8 & 3430.2 \\
\hline 3141.7 & 2978.4 & & 3143.0 & 2979.0 \\
\hline 3126.3 & 2968.9 & & 3125.0 & 2967.5 \\
\hline 1813.8 & 1778.1 & C=O out of phase & 1813.1 & 1778.5 \\
\hline 1789.9 & 1757.0 & C=O in-phase & 1783.9 & 1751.0 \\
\hline 1445.6 & 1399.3 & & 1449.1 & 1398.9 \\
\hline 1427.7 & 1388.8 & & 1427.1 & 1391.1 \\
\hline 1358.3 & 1305.9 & & 1369.0 & 1317.0 \\
\hline 1298.9 & 1257.0 & & 1301.1 & 1255.7 \\
\hline $\mathbf{1 1 8 7 . 6}$ & $\mathbf{1 1 5 0 . 3}$ & C-O(-H) donor & $\mathbf{1 1 9 4 . 6}$ & $\mathbf{1 1 5 7 . 6}$ \\
\hline $\mathbf{1 1 4 3 . 5}$ & $\mathbf{1 1 0 9 . 9}$ & C-O(-H) acceptor & $\mathbf{1 1 4 1 . 1}$ & $\mathbf{1 1 0 6 . 4}$ \\
\hline 671.3 & 657.7 & & 677.9 & 664.5 \\
\hline 645.1 & 636.1 & & 648.9 & 640.4 \\
\hline 192.3 & 174.3 & & 217.4 & 198.7 \\
\hline 110.6 & 98.1 & & 172.9 & 151.0 \\
\hline 61.7 & 62.7 & & 32.8 & 26.6 \\
\hline & & & & \\
\hline 1075.6 & 1051.0 & & 1078.5 & 1052.5 \\
\hline 1063.6 & 1041.5 & & 1065.0 & 1040.6 \\
\hline $\mathbf{8 0 1 . 2}$ & $\mathbf{7 5 0 . 6}$ & H out of plane & $\mathbf{8 2 8 . 5}$ & $\mathbf{7 7 0 . 8}$ \\
\hline $\mathbf{7 2 3 . 2}$ & $\mathbf{6 7 1 . 7}$ & H out of plane & $\mathbf{7 3 9 . 7}$ & $\mathbf{6 8 1 . 4}$ \\
\hline 162.1 & 143.4 & & 141.8 & 126.9 \\
\hline 133.6 & 119.4 & & 124.2 & 110.0 \\
\hline 28.4 & 22.2 & & 67.2 & 66.0 \\
\hline
\end{tabular}

Italic $=$ intense IR absorbers

Table S6: selected harmonic frequencies $\left(\mathrm{cm}^{-1}\right)$ species VI-VIII

\begin{tabular}{|l|l|l|l|}
\hline System & Species VI & Species VII & Species VIII \\
\hline Frequencies & & & \\
OCO & 636,631 & $632,(631)$ & $636,(634)$ \\
COH out of plane & 678,666 & $669,(664)$ & $679,(676)$ \\
OCH out of plane & 1079,1077 & $1072,(1072)$ & $1083,(1085)$ \\
CO stretch & 1127,1149 & $1139,(1123)$ & $1134,(1154)$ \\
COH in plane & 1317,1300 & $1300,(1295)$ & $1314,(1316)$ \\
\hline
\end{tabular}


Table S7: III vs III'

\begin{tabular}{|l|c|c|c|c|c|}
\hline & \multicolumn{2}{l|}{ Trans-Trans III } & & \multicolumn{2}{l|}{ Cis-trans III' } \\
\hline & & & & & \\
\hline $1(1)$ & 3788.875 & 3599.912 & $1(1)$ & 3857.726 & 3673.506 \\
\hline $2(1)$ & 3515.020 & 3320.087 & $2(1)$ & 3492.809 & 3295.918 \\
\hline $3(1)$ & 3180.855 & 3031.787 & $3(1)$ & 3119.365 & 2961.620 \\
\hline $4(1)$ & 3119.150 & 2962.953 & $4(1)$ & 3105.671 & 2958.832 \\
\hline $\mathbf{5 ( 1 )}$ & $\mathbf{1 7 9 5 . 2 0 7}$ & $\mathbf{1 7 6 3 . 1 6 0}$ & $\mathbf{5 ( 1 )}$ & $\mathbf{1 8 1 6 . 3 0 8}$ & $\mathbf{1 7 7 8 . 3 7 2}$ \\
\hline $\mathbf{6 ( 1 )}$ & $\mathbf{1 7 6 3 . 7 6 3}$ & $\mathbf{1 7 3 5 . 4 9 9}$ & $\mathbf{6 ( 1 )}$ & $\mathbf{1 7 7 5 . 8 4 4}$ & $\mathbf{1 7 5 3 . 6 4 6}$ \\
\hline $7(1)$ & 1456.442 & 1406.445 & $7(1)$ & 1456.885 & 1409.111 \\
\hline $8(1)$ & 1424.999 & 1405.520 & $8(1)$ & 1452.189 & 1415.836 \\
\hline $9(1)$ & 1392.405 & 1348.716 & $9(1)$ & 1393.548 & 1344.617 \\
\hline $10(1)$ & 1335.889 & 1292.894 & $10(1)$ & 1309.467 & 1263.566 \\
\hline $\mathbf{1 1 ( 1 )}$ & $\mathbf{1 2 1 6 . 5 1 5}$ & $\mathbf{1 1 7 8 . 7 1 3}$ & $\mathbf{1 1}(\mathbf{1})$ & $\mathbf{1 2 2 2 . 1 0 5}$ & $\mathbf{1 1 8 4 . 1 4 1}$ \\
\hline $\mathbf{1 2 ( 1 )}$ & $\mathbf{1 1 7 3 . 5 0 3}$ & $\mathbf{1 1 3 9 . 1 2 4}$ & $\mathbf{1 2}(\mathbf{1})$ & $\mathbf{1 1 5 5 . 1 8 5}$ & $\mathbf{1 1 2 7 . 7 6 8}$ \\
\hline $13(1)$ & 679.890 & 667.193 & $13(1)$ & 675.419 & 667.646 \\
\hline $14(1)$ & 652.520 & 644.490 & $14(1)$ & 542.558 & 525.413 \\
\hline $15(1)$ & 174.710 & 158.981 & $15(1)$ & 176.017 & 161.994 \\
\hline $16(1)$ & 144.297 & 137.442 & $16(1)$ & 144.882 & 136.635 \\
\hline $17(1)$ & 103.725 & 93.978 & $17(1)$ & 110.454 & 99.670 \\
\hline & & & & & \\
\hline $18(1)$ & 1097.780 & 1070.425 & $18(1)$ & 1082.904 & 1060.286 \\
\hline $19(1)$ & 1080.841 & 1057.573 & $19(1)$ & 1080.816 & 1052.345 \\
\hline $20(1)$ & $\mathbf{8 7 7 . 0 4 4}$ & $\mathbf{8 5 7 . 9 8 7}$ & $20(1)$ & $\mathbf{8 7 8 . 8 1 9}$ & $\mathbf{8 7 4 . 4 3 6}$ \\
\hline $21(1)$ & $\mathbf{6 8 8 . 5 3 6}$ & $\mathbf{6 3 7 . 6 1 5}$ & $21(1)$ & $\mathbf{6 8 4 . 5 9 7}$ & $\mathbf{6 7 3 . 2 0 0}$ \\
\hline $22(1)$ & 181.880 & 178.922 & $22(1)$ & 183.174 & 184.269 \\
\hline $23(1)$ & 87.946 & 83.869 & $23(1)$ & 95.884 & 94.726 \\
\hline $24(1)$ & 54.847 & 56.913 & $24(1)$ & 57.296 & 58.981 \\
\hline & & & & & \\
\hline & & & & & \\
\hline & & & & & \\
\hline
\end{tabular}


Table S8: Energy and structure of Transition States for interconversions leading to II

\begin{tabular}{|c|c|c|c|}
\hline Species & $\mathrm{V} \rightarrow \mathrm{II}$ & III $\rightarrow$ II & OPEN $\rightarrow$ II \\
\hline \multicolumn{4}{|l|}{ Structure } \\
\hline $\mathrm{C}=\mathrm{O} \quad(\mathrm{Y}-\mathrm{T})$ & $1.2104,1.2116$ & $1.2092,1.2099$ & $1.2061,1.2144$ \\
\hline $\mathrm{C}=\mathrm{O} \quad(\mathrm{BTYS})$ & & $1.211,1.212$ & \\
\hline $\mathrm{C}-\mathrm{O} \quad(\mathrm{Y}-\mathrm{T})$ & $1.3382,1.3340$ & $1.3394,1.3366$ & $1.3589,1.3306$ \\
\hline $\mathrm{C}-\mathrm{O} \quad$ (BTYS) & & $1.340,1.336$ & \\
\hline $\mathrm{OH} \quad(\mathrm{Y}-\mathrm{T})$ & $0.9761,9696$ & $0.9746,0.9695$ & $0.9701,0.9771$ \\
\hline $\mathrm{OH} \quad(\mathrm{BTYS})$ & & $0.977,0.969$ & \\
\hline $\mathrm{CH} \quad(\mathrm{Y}-\mathrm{T})$ & $1.0974,1.0949$ & $1.0973,1.0949$ & $1.0990,1.0958$ \\
\hline CH $\quad$ (BTYS) & & $1.091,1.088$ & \\
\hline$-\mathrm{OH} \ldots \mathrm{O}=(\mathrm{Y}-\mathrm{T})$ & $1.8803,3.5968$ & $1.9634,3.9118$ & $2.0074,2.1179$ \\
\hline$-\mathrm{OH} \ldots \mathrm{O}=(\mathrm{BTYS})$ & & 1.847 & \\
\hline Barrier from II (w. ZPE) & $5.27(4.33)$ & $6.80(5.76)$ & $15.61(13.85)$ \\
\hline Reverse Barrier (w. ZPE) & $0.71(0.52)$ & $2.26(1.77)$ & $5.08(4.39)$ \\
\hline $\mathrm{E}$ (hartrees) & -378.7348638 & -378.7324325 & -378.7183938 \\
\hline $\mathrm{ZPE}$ & 43.856 & 43.759 & 43.033 \\
\hline
\end{tabular}




\section{Calculated and Experimental Frequencies for Acetic Acid's C2h Dimer}

\begin{tabular}{|c|c|c|c|c|c|c|c|c|c|c|}
\hline & & CP & $f(C P)$ & MP2(II) & f(MP2) & Scaled & EXP & Barone & MP2* & $f\left(M^{\prime} 2^{*}\right)$ \\
\hline $\mathrm{Bu}$ & $\mathrm{OH}$ str & 3495 & 2056 & 3357 & 2338 & 3199.2 & 2965 & 3119 & 3386 & 2647 \\
\hline Ag & & 3436 & 0 & 3276 & 0 & 3122.0 & & 3022 & 3303 & \\
\hline $\mathrm{Bu}$ & $\mathrm{CH}$ & 3226 & 8 & 3257 & 10 & 3103.9 & 3035 & 3081 & 3226 & 9 \\
\hline $\mathbf{A g}$ & & 3226 & 0 & 3256 & 0 & 3103.0 & & 3078 & 3226 & \\
\hline $\mathrm{Au}$ & & 3187 & 4 & 3217 & 4 & 3065.8 & & 3056 & 3186 & 4 \\
\hline $\mathrm{Bg}$ & & 3187 & 0 & 3217 & 0 & 3065.8 & & 3056 & 3186 & \\
\hline $\mathrm{Bu}$ & & 3101 & 0 & 3131 & 1 & 2983.8 & & 2991 & 3101 & 3 \\
\hline Ag & & 3101 & 3 & 3131 & 0 & 2983.8 & & 2991 & 3101 & \\
\hline $\mathrm{Bu}$ & $C=0$ & 1796 & 733 & 1825 & 625 & 1739.2 & 1737 & 1747 & 1789 & 746 \\
\hline Ag & $C=0$ & 1761 & 0 & 1789 & 0 & 1704.9 & 1682 & 1735 & 1747 & \\
\hline $\mathbf{A g}$ & Me & 1495 & 0 & 1538 & 0 & 1465.7 & 1428 & 1440 & 1503 & \\
\hline $\mathrm{Au}$ & Me & 1495 & 19 & 1538 & 19 & 1465.7 & & 1453 & 1495 & 19 \\
\hline $\mathrm{Bu}$ & $\mathrm{Me}$ & 1492 & 46 & 1535 & 52 & 1462.9 & 1430 & 1444 & 1493 & 52 \\
\hline $\mathrm{Bg}$ & Me & 1494 & 0 & 1540 & 0 & 1467.6 & & 1452 & 1495 & \\
\hline $\mathrm{Bu}$ & & 1461 & 208 & 1505 & 209 & 1434.23 & 1430 & 1415 & 1471 & 162 \\
\hline $\mathbf{A g}$ & & 1464 & 0 & 1516 & & 1444.7 & 1428 & 1424 & 1482 & \\
\hline $\mathbf{A g}$ & & 1406 & 0 & 1448 & & 1379.9 & 1370 & 1372 & 1414 & \\
\hline $\mathrm{Bu}$ & & 1396 & 5 & 1436 & 1 & 1368.5 & & 1365 & 1406 & 47 \\
\hline $\mathrm{Bu}$ & & 1319 & 394 & 1350 & 365 & 1286.6 & 1290 & 1298 & 1339 & 395 \\
\hline Ag & & 1307 & 0 & 1342 & & 1278.9 & 1285 & 1281 & 1332 & \\
\hline $\mathrm{Au}$ & & 1077 & 11 & 1108 & 11 & 1055.9 & 1066 & 1072 & 1076 & 9 \\
\hline $\mathrm{Bg}$ & & 1076 & 0 & 1107 & 0 & 1055.0 & 1065 & 1078 & 1070 & \\
\hline $\mathrm{Bu}$ & & 1040 & 49 & 1066 & 37 & 1016.0 & 1014 & 1042 & 1042 & 43 \\
\hline $\mathbf{A g}$ & & 1038 & 0 & 1064 & 0 & 1014.0 & 1007 & 1025 & 1040 & \\
\hline $\mathbf{A g}$ & & 911 & 0 & 925 & 0 & 881.5 & 891 & 900 & 916 & \\
\hline $\mathrm{Bu}$ & & 910 & 6 & 924 & 4 & 880.6 & 896 & 900 & 915 & 6 \\
\hline $\mathrm{Au}$ & & 905 & 201 & 987 & 254 & 940.6 & 942 & 1127 & 911 & 218 \\
\hline $\mathrm{Bg}$ & & 864 & 0 & 941 & & 896.8 & & 1119 & 868 & \\
\hline $\mathrm{Bu}$ & & 624 & 53 & 632 & 54 & 602.3 & 623 & 627 & 634 & 50 \\
\hline $\mathbf{A g}$ & & 620 & 0 & 627 & 0 & 597.5 & 616 & 621 & 627 & \\
\hline $\mathrm{Bg}$ & & 599 & 0 & 610 & 0 & 581.3 & 616 & 660 & 576 & \\
\hline $\mathrm{Au}$ & & 592 & 1 & 601 & 1 & 572.8 & & 596 & 590 & \\
\hline $\mathrm{Bu}$ & & 466 & 29 & 483 & 34 & 460.3 & & 474 & 476 & 38 \\
\hline $\mathbf{A g}$ & & 443 & 0 & 446 & 0 & 425.0 & 439 & 441 & 445 & \\
\hline $\mathrm{Au}$ & Methyl & 33 & & 87 & $<1$ & 82.9 & & 533 & 20 & 2 \\
\hline $\mathrm{Bg}$ & & 30 & & 80 & 0 & 76.2 & & 602 & 19 & \\
\hline \multicolumn{11}{|c|}{ INTERMOL } \\
\hline $\mathrm{Bu}$ & & 147 & 24 & 174 & 23 & 165.8 & 171 & 171 & 166 & 28 \\
\hline $\mathbf{A g}$ & & 152 & 0 & 178 & 0 & 169.6 & 155 & 157 & 166 & \\
\hline $\mathbf{A g}$ & & 140 & 0 & 157 & & 149.6 & 120 & 140 & 154 & \\
\hline $\mathrm{Bg}$ & & 111 & 0 & 128 & & 122.0 & 99 & 136 & 103 & \\
\hline $\mathrm{Au}$ & & 68 & 1 & 51 & 4 & 48.6 & 56 & 127 & 37 & 2 \\
\hline $\mathrm{Au}$ & twist & 56 & 0 & 80 & & 76.2 & 48 & 86 & 62 & $<1$ \\
\hline
\end{tabular}

